vol. $27-n^{\circ} 3 \mid 2011$

Numéro ouvert

\title{
Yasmina BETTAHAR et Françoise BIRK, Étudiants étrangers en France. L'émergence de nouveaux pôles d'attraction au début du XXe siècle
}

\section{Ralph Schor}

\section{(2) OpenEdition \\ Journals}

Édition électronique

URL : https://journals.openedition.org/remi/5579

DOI : $10.4000 /$ remi.5579

ISSN : $1777-5418$

Éditeur

Université de Poitiers

Édition imprimée

Date de publication : 1 décembre 2011

Pagination : 202-204

ISBN : 979-10-90426-02-3

ISSN : 0765-0752

Référence électronique

Ralph Schor, « Yasmina BETTAHAR et Françoise BIRK, Étudiants étrangers en France. L'émergence de nouveaux pôles d'attraction au début du XXe siècle », Revue européenne des migrations internationales [En ligne], vol. 27 - n³ | 2011, mis en ligne le 17 avril 2012, consulté le 15 avril 2022. URL : http:// journals.openedition.org/remi/5579; DOI : https://doi.org/10.4000/remi.5579 


\section{NOTES DE LECTURE}

LAACHER, Smaïn

De la violence à la persécution, femmes sur les routes de l'exil. - Paris : La dispute, 2010. - $170 \mathrm{p}$.

ISBN : 978-2-84303-197-7

\section{Des violences en chemin, dévoiler et rendre compte $^{1}$}

C'est à une opération de dévoilement salutaire que Smaïn Laacher nous convie dans son dernier ouvrage, De la violence à la persécution, femmes sur les routes de l'exil. À la fois au sens de lever le voile, en rendant public un phénomène connu des acteurs de l'aide aux réfugiés comme des chercheurs - à savoir que parmi les exactions commises à l'encontre des migrants traversant le Sahara, certaines touchent plus particulièrement les femmes du fait même de leur genre : viols, prostitution forcée, violences à caractère sexuel... mais qui ne donnait jusqu'à présent pas matière à développement autre que des mentions, des allusions ou des notes de bas de page, comme si les violences envers les femmes sur les routes migratoires étaient un dommage collatéral dont ils ne savaient pas bien comment rendre compte, a fortiori s'ils en avaient été les témoins impuissants; des souffrances de plus parmi tant d'autres.

$E t$, dévoilement au sens de dire, voire de tenter d'expliquer, l'indicible. Indicible parce qu'il s'agit d'actes de violence dont l'expérience est vécue comme incommensurable et honteuse ; certaines parlent de "souillure" qui les met irrémédiablement à l'écart et les condamne. Indicible parce que leur restitution engage la question de la mémoire, mémoire fragmentaire qui fait parfois défaut aux victimes dont le récit est parcellaire, faute de repères spatio-temporels précis durant un voyage

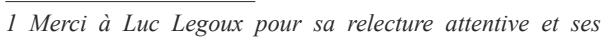
suggestions amicales. souvent long, toujours éprouvant et chaotique ${ }^{2}$. Indicible parce que l'acte même de témoigner conditionne en partie l'issue (dé)favorable de leur demande d'asile, de prise en charge ou d'assistance et induit donc une mise en discours conditionnée par les attentes supposées des institutions. Autant d'éléments que Smaïn Laacher s'est attaché à dénouer afin de rendre compte de réalités, parfois insoutenables, dont font état la soixantaine de migrantes africaines qu'il a rencontrées dans des structures d'aide ou d'accueil en France, en Espagne, mais aussi au Maroc ou en Algérie.

Ce choix dont on comprend qu'il est le plus rentable pour recueillir leur témoignage est cependant en deçà des objectifs d'un projet qui prétend rendre compte des violences "pendant le voyage ". Celui-ci aurait mérité de croiser différentes approches et de ne pas s'en tenir au recueil d'entretiens dont l'auteur souligne lui-même les limites et les difficultés. Ainsi, l'immersion dans les villes et les oasis de transit $t^{3}$ aurait, par exemple, permis de mieux comprendre les facteurs de vulnérabilité et les rapports de domination dans leur quotidienneté. Cela aurait aussi permis de prendre du temps avec ces femmes et de laisser une place aux silences. C'est d'ailleurs peut-être sous l'angle de la pudeur et du respect que l'on aurait souhaité voir aborder la question de l'éthique de l'enquête plus que sous celui de

\footnotetext{
2 Cette question est bien connue et documentée notamment en histoire. Ainsi Daniel Cordier, historien et acteur de la résistance, note " je compris alors que les images précises qui m'avaient hanté n'étaient que des lambeaux d'une réalité mutilée. [...] Le néant circonscrit les morceaux irréguliers et précis du réel comme une mer noire emprisonne des récifs déchiquetés. Il en est de même pour mon passé. J'ai beau écarquiller les yeux, scruter ces images si denses dans leur détail, sans qu'il soit possible d'en découvrir les prolongements et les articulations logiques " (CORDIER Daniel et GUILLON Jean-Marie Guillon (1995) Mémoire et Histoire : la Résistance, Toulouse, Privat, pp. 308-309).

3 Sur cette question du trajet comme terrain, voir BRACHET Julien (2008) Géographie du mouvement, géographie en mouvement. La mobilité comme dimension du terrain dans l'étude des migrations, in À travers l'espace de la méthode : les dimensions du terrain en géographie [en ligne]. URL: http://hal.archives-ouvertes.fr/halshs-00358932/
} 
l'anonymat et du consentement éclairé qui en l'état relève d'une éthique de charte plus que d'une éthique en situation. Enfin, la mobilisation des études sur le genre $e^{4}$ et sur la violence aurait certainement enrichi l'analyse en différenciant mieux les logiques à l'œuvre et en évitant de réifier les différences entre les sexes. À ce titre, les mentions qui sont faites des hommes violés, de manière isolée ou collective, comme des violences perpétrées par des migrantes à l'encontre d'autres femmes jugées déviantes, ouvrent des pistes intéressantes pour aborder des phénomènes complexes.

Cependant, ces critiques sont à replacer dans le contexte d'élaboration de cet ouvrage. Tout d'abord, saluons le courage de l'auteur d'avoir traité d'un sujet difficile - les violences envers les femmes en contexte migratoire - qui est fortement genré ; ce sujet étant plus fréquemment l'apanage des chercheuses. Rappelons ensuite que l'auteur est mandaté par le HCR. Il vise donc la prise en compte de ces violences par les institutions nationales et internationales et la mise en place de politiques de protections qui dépassent le cadre originel du statut de réfugié. Le statut est en effet conçu pour offrir une protection à ceux que leur État d'origine persécute ou est incapable de protéger contre des persécutions commises sur leur territoire. Dans le cas des migrantes le défaut de protection des pays d'origine ne peut être évoqué, mais si la protection des migrants, femmes et hommes, n'est pas directement $d u$ ressort $d u$ statut de réfugié, il n'en reste pas moins qu'une réflexion globale sur la cause des migrations et la protection dans les pays de transit est nécessaire, elle est malheureusement à peine esquissée dans ce texte qui a cependant le mérite de poser le problème. Smaïn Laacher vise en effet l'efficacité de l'action dans un temps - celui de l'enquête et de l'analyse réduit. Or, si alerter et mettre les décideurs

4 Notamment les travaux de Paola Tabet et la notion d'échange économico-sexuel. Pour une première approche de ses travaux, voir TRACHMAN Mathieu (2009) La banalité de l'échange. Entretien avec Paola Tabet, Genre, sexualité \& société, 2 [en ligne]. URL : http://gss.revues.org/index1227. html

5 Pour exemple, l'ouvrage de SOFSKY Wolfgang (1998) Traité de la violence, Paris, Gallimard, $214 p$. face à leurs responsabilités - a fortiori s'ils instrumentalisent la question des rapports de genre à des fins politiques - est une nécessité, pour autant ce sujet mériterait de faire l'objet d'un travail d'enquête approfondi et ambitieux qui articule le champ des migrations, celui du genre et celui de la violence. Espérons que cet ouvrage en constitue les prémices.

Marianne Blidon

MCF IDUP

BETTAHAR, Yasmina (dir.)
BIRK, Françoise (dir.)

Étudiants étrangers en France. L'émergence de nouveaux pôles d'attraction au début du XXe siècle. - Nancy : Presses universitaires de Nancy, 2009. - $130 \mathrm{p}$.

ISBN : 978-2-86480-976-0

Tes pays d'Europe occidentale et particulièrement la France accueillent des étudiants étrangers dont le nombre a considérablement augmenté depuis le début du $X X e$ siècle. Au début, les jeunes viennent surtout d'Europe centrale et balkanique, puis les Africains prennent le relais. Les contingents les plus nombreux se dirigent vers Paris dont le prestige reste sans égal. Cependant les universités de province ne sont pas négligées. Un colloque organisé à Nancy en décembre 2002 $a$ attiré l'attention sur ce phénomène, surtout dans le domaine des formations scientifiques et techniques.

Les auteurs rappellent d'abord que l'arrivée des étudiants étrangers est due en grande partie, au XIXe siècle, aux politiques restrictives mises en place par certains États qui limitent l'accès de leurs facultés selon des critères politiques ou ethnico-religieux, tout particulièrement au détriment des Juifs. Quant aux autorités françaises, elles souhaitent attirer des étrangers pour concurrencer l'attraction ancienne exercée par les universités allemandes. Autre préoccupation constante et complémentaire : former des cadres allogènes "à la française " pour accroître le rayonnement $d u$ pays d'accueil, amener les anciens étudiants à rester fidèles aux méthodes, aux idées, aux produits, 
aux brevets découverts en France. Le décret du 21 juillet 1897 distingue les diplômes d'État et les diplômes d'université. Ces derniers sont plus particulièrement destinés aux étrangers et ferment en principe certaines professions libérales aux non-citoyens, ce qui satisfait les nationaux toujours portés à dénoncer la concurrence étrangère en temps de crise. Cette clarification des diplômes permet aux établissements de diversifier leur offre de formation.

L'existence de ces cadres générauxn'empêche pas une certaine diversité, analysée à travers quelques exemples. Le premier cas étudié est celui des Roumains dans les écoles militaires françaises. Depuis le début du XIXe siècle, de nombreux jeunes venus de Moldavie et de Valachie viennent étudier à Paris et fournissent les têtes pensantes du mouvement d'unification nationale. Celle-ci accomplie, les dirigeants restent fidèles à l'influence française, mais ils se heurtent à la famille royale, d'origine allemande, tournée vers les institutions germaniques. Les attachés militaires français à Bucarest ne cessent de rappeler qu'il faut faciliter l'accueil des Roumains dans les écoles militaires de l'hexagone, ce qui doit garantir la francophilie politique des futurs officiers. Mais les autorités françaises hésitent, car elles sont sensibles à l'argument politique, mais elles craignent aussi de laisser échapper des secrets liés à la défense nationale.

L'Institut électrotechnique de Nancy, fondé en 1900, et l'université de Strasbourg, redevenue française en 1919, offrent des points communs : ce sont des établissements proches de la frontière qui tiennent ainsi un rôle symbolique de môles de la culture française face à l'Allemagne qu'il faut concurrencer sur son propre terrain. Les industries régionales veulent détourner les jeunes nationaux de se former de l'autre côté du Rhin. À Strasbourg les locaux universitaires sont très vastes et doivent accueillir des étudiants nombreux pour amortir les frais généraux. Or l'anémie démographique de la France éclaircit les effectifs d'étudiants. Aussi les établissements essaient d'attirer de gros effectifs d'étudiants, leur assurent diverses facilités, des locaux fonctionnels, des forma- tions adaptées et une large gamme de diplômes. Le succès vient rapidement et les universités frontalières reçoivent jusqu'à un tiers d'étrangers. Mais la crise des années 1930 amène les Français, hantés par la peur de la concurrence, à rendre des attitudes xénophobes et antisémites, ce qui entraîne un reflux des effectifs étrangers.

L'Institut polytechnique de l'Ouest est ouvert à Nantes en 1919 pour fournir des techniciens aux industries locales. La présence des étrangers est attestée à l'Institut, mais n'est pas particulièrement recherchée par la direction. Les effectifs d'étrangers baissent dans les années 1930, sans doute pour les mêmes raisons que dans l'Est.

L'Institut chimique de Rouen, ouvert en 1917, doit lui aussi répondre aux besoins de l'économie de la région. Les industriels locaux, marqués par le saint-simonisme, se montrent favorables à une ouverture démocratique de l'enseignement supérieur technique et, pour marquer la place de l'Institut, acceptent la venue de nombreux étrangers - 55,7\% des inscrits dans l'année 1929-1930 - principalement juifs. La direction facilite la vie quotidienne et le placement des élèves dont un nombre non négligeable essaie de rester en France après la fin des études.

Depuis les années 1960-1970, les écoles d'ingénieurs françaises, dont l'École nationale supérieure d'électricité et de mécanique de Nancy, attirent de nombreux Maghrébins, notamment des Marocains dont l'exemple est ici analysé. Ces étudiants sont surtout issus des milieux de notables traditionnels, avec une tendance à l'extension vers les classes moyennes. Leur venue est organisée dans le cadre d'accords de coopération interétatique. Les diplomés trouvent généralement des postes de responsabilité dans leur pays ou s'orientent vers l'international. Dans les deux cas, ils semblent fidèles au modèle français dont ils assurent le rayonnement.

Cet ouvrage est bien documenté, dans la mesure où les auteurs ont trouvé des archives 
explicites. Quand ce n'est pas le cas, ils avancent avec prudence des hypothèses vraisemblables. Ils présentent parfois, à titre d'exemple probant, les parcours universitaire et professionnel de certains étrangers dont les dossiers personnels existent encore. L'histoire des établissements étudiés est toujours clairement replacée dans l'évolution générale. Le livre souligne la diversité du réel : selon les établissements, la venue des jeunes étrangers a été activement recherchée ou s'est effectuée à son rythme propre. De même, les relations entre les autochtones et leurs camarades venus de loin ont pu rester neutres ou subir des dérapages xénophobes qui ont infléchi le recrutement des élèves. Ainsi cet ouvrage original constitue une utile contribution à plusieurs chapitres de l'histoire : ceux de la diplomatie, de l'enseignement supérieur, de l'économie régionale, des migrations intellectuelles, des relations interethniques.

Ralph Schor Département d'Histoire Université de Nice-Sophia-Antipolis 